\title{
BMJ Open Cross-sectional study of HPV testing in self-sampled urine and comparison with matched vaginal and cervical samples in women attending colposcopy for the management of abnormal cervical screening
}

\author{
Alex Sargent, ${ }^{1}$ Samantha Fletcher, ${ }^{2}$ Katarina Bray, ${ }^{2}$ Henry C Kitchener, ${ }^{2}$ \\ Emma J Crosbie ${ }^{\oplus 2,3}$
}

To cite: Sargent A, Fletcher $S$, Bray K, et al. Cross-sectional study of HPV testing in selfsampled urine and comparison with matched vaginal and cervical samples in women attending colposcopy for the management of abnormal cervical screening. BMJ Open 2019;9:e025388. doi:10.1136/ bmjopen-2018-025388

- Prepublication history and additional material for this paper are available online. To view these files, please visit the journal online (http://dx.doi. org/10.1136/bmjopen-2018025388).

Received 12 July 2018 Revised 3 January 2019 Accepted 4 February 2019

D) Check for updates

(C) Author(s) (or their employer(s)) 2019. Re-use permitted under CC BY-NC. No commercial re-use. See rights and permissions. Published by BMJ.

For numbered affiliations see end of article.

Correspondence to Dr Emma J Crosbie; emma.crosbie@manchester. ac.uk

\section{ABSTRACT}

Objectives Human papillomavirus (HPV) testing in cervical screening offers the potential for self-sampling to improve uptake among non-attenders. High-risk (HR) HPV detection in urine shows promise, but few studies have examined its sensitivity for cervical intraepithelial neoplasia (CIN2+) detection compared with standard cervical samples. The aims of this cross-sectional study were to optimise conditions for urine testing for HPV detection; to determine concordance for HR-HPV detection in matched urine, vaginal and cervical samples; to compare the sensitivity of HR-HPV testing for the detection of CIN2+ in matched samples; and to determine the acceptability of urine testing for cervical screening.

Design Cross-sectional study.

Setting Secondary care colposcopy clinic in North West England.

Participants Women aged 25 years of age or older, attending colposcopy clinic for management of abnormal cervical screening results or a suspicious-looking cervix. In total, 104 women took part in the study. Triple matched samples were available for 79 and 66 women using Abbott RealTime (ART) and Roche Cobas 4800 (RC), respectively. Intervention Self-collected urine and vaginal samples and practitioner-obtained cervical samples were tested for HR-HPV by ART and RC assays, including comparison of neat and preservative-fixed urine. Colposcopic opinion was recorded and directed cervical biopsies taken if clinically indicated. The acceptability of self-testing was evaluated by questionnaire.

Primary outcome measure The sensitivity of urine to detect underlying CIN2+.

Secondary outcome measures The comparative sensitivity of vaginal and cervical samples to detect CIN2+; the acceptability of urine sampling.

Results Preservative-fixed, but not neat urine, showed good concordance with vaginal samples for the detection of HR-HPV. The sensitivity for detecting CIN2+ was 15/18 (83\%) for urine and 16/18 (89\%) for cervical and vaginal samples by ART, and 15/17 (88\%) for all samples by RC. Urine-based testing was broadly acceptable to women.
Strengths and limitations of this study

- We studied the clinical performance of urine human papillomavirus (HPV) testing in colposcopy clinic attendees, not cervical screening non-attenders, in whom the technology is likely to have most value.

- We compared neat and preservative-fixed urine using two different high-risk (HR)-HPV detection assays alongside matched vaginal and cervical samples.

- The virologist was blinded to clinical outcomes at the time of HR-HPV testing.

- The clinical performance of urine HPV testing may be affected by the prevalence of HR-HPV in the population, which is higher in colposcopy clinic attendees than cervical screening non-attenders.

- We determined the acceptability of urine HPV testing by studying women's preferences for urine, vaginal and cervical sampling, while acknowledging that cervical screening attenders may have different preferences to non-attenders.

Conclusions Urinary HR-HPV detection offers an alternative strategy of cervical screening. Larger studies to determine its clinical utility are warranted.

\section{BACKGROUND}

The strategy of cervical screening depends on reducing the incidence of cervical cancer through the detection and treatment of its precursor lesion, high grade cervical intraepithelial neoplasia (CIN2+). ${ }^{1}$ Cervical cancer is caused by persistent infection with highrisk human papillomavirus (HR-HPV) ${ }^{2}$ and a pooled analysis of four large randomised controlled trials (RCTs) found that detection of HR-HPV in practitioner-obtained cervical samples is a more sensitive primary screen 
than cervical cytology for the detection of CIN2+. ${ }^{3}$ The UK National Screening Committee, together with other publically funded programmes, has therefore recommended moving to primary HPV testing for general population screening, with reflex cytology on positive samples.

Despite the success of cervical screening, recent years have witnessed a gradual decline in its uptake, particularly by women aged 25-49 years. Barriers to cervical screening include embarrassment, fear, inconvenience and some women believe that they are not at risk. ${ }^{4}$ It may be possible to overcome some of these barriers by improving the acceptability of the screening test and the ease with which it can be performed. Vaginal self-sampling kits for HR-HPV detection have a modest impact on screening uptake by chronic non-attenders. ${ }^{56}$ The diagnostic accuracy of HPV detection in self-obtained vaginal samples has similar accuracy to practitioner-obtained cervical samples, and is sufficient to recommend its use in non-attenders and in low resource settings. ${ }^{7}$

HPV detection in urine has recently been considered as an alternative means to identify women at increased risk of high grade CIN. A systematic review with meta-analysis found a pooled sensitivity of $77 \%$ and specificity of $88 \%$ for HR-HPV detection in urine. ${ }^{89}$ This pooled sensitivity was lower than for HR-HPV detection in practitioner-obtained cervical samples but the wide variation in methodology, including urine sampling techniques, HPV detection methodology and study setting precluded unbiased comparison. Since HPV testing is more sensitive than cytology for the detection of CIN2+, a degree of decreased sensitivity of urinary HPV detection could be offset by improved uptake of cervical screening. ${ }^{10}$

The aims of this exploratory study were fourfold: to optimise urine processing for HR-HPV detection; to establish the concordance of HR-HPV detection in matched urine, vaginal and cervical samples in a colposcopy population; to compare the sensitivity of HR-HPV testing for CIN2+ detection in the same samples; and to determine the acceptability by women of self-collected versus practitioner-obtained samples for cervical screening.

\section{METHODS}

\section{Study participants and sample collection}

Urine, vaginal and cervical samples were collected from consecutive women attending the colposcopy clinic at St Mary's Hospital, Manchester University NHS Foundation Trust (MFT) between June and September 2015. A participant information sheet was sent with the colposcopy appointment letter to women who were 25 years of age or older and attending the colposcopy clinic for management of abnormal cervical screening results or a suspicious-looking cervix. All women gave written, informed consent to take part. Women who declined participation in the study were asked to give their reasons. Pregnant women were excluded from the study. Study samples were obtained prior to colposcopic examination of the cervix, where this was required. Self-collected first stream urine samples were collected in a sterile dry pot, followed by self-collected vaginal samples obtained using the Rovers Evalyn Brush (Netherlands). Women were asked to place the brush in the vagina, rotate it five times for sample collection and replace the brush in its casing. Practitioner-obtained cervical samples were then taken with a cervical broom immediately prior to clinically indicated procedures. Following colposcopy, women were asked to complete a questionnaire about the acceptability of the tests performed (online supplementary appendix 1).

\section{Clinical procedures}

Women were managed clinically according to National Health Service (NHS) Cervical Screening Programme guidelines. All colposcopists were accredited for independent practice by the British Society of Colposcopy and Cervical Pathology (BSCCP). Abnormal colposcopy indicated directed punch biopsy and histological review by consultant gynaecological histopathologists, who classified biopsies as normal, low grade CIN (CIN1), or high grade CIN (CIN2/3).

\section{HPV DNA testing}

Blinded samples were delivered to the MFT Virology laboratory within 4 hours of collection. Cervical samples were collected using SurePath Liquid Based Cytology and vaginal cells were suspended in solution by agitating the Evalyn brush in SurePath preservative on receipt in the laboratory. Sample aliquots were added to Abbott and Roche secondary tubes as per the manufacturers' instructions. Batches of samples were tested for high risk HPV using the Abbott RealTime (ART) and Roche Cobas 4800 (RC) assays within 48 hours and 1 week of sample processing, respectively. Both assays used fully automated systems that amplify and detect 14 HR-HPV genotypes $(16,18,31,33,35,39,45,51,52,56,58,59$, 66 and 68) simultaneously. Separate probes enable the specific detection of HPV types 16 and 18. Both assays also measure human beta globin as a control for sample cellularity, valid sample extraction and amplification. The cycle threshold (ct) is a measure of DNA concentration, with ct values inversely proportional to the amount of target nucleic acid in the sample. All samples were tested according to the manufacturers' instructions. Both assays (ART and RC) were applied to all samples, where sufficient material was available.

\section{Optimisation of urine sample processing for HR-HPV testing}

Urine sample processing was optimised by comparing HR-HPV detection in samples refrigerated at $4^{\circ} \mathrm{C}$ both without preservative ('neat') and following transfer to Abbott and Roche preservative tubes ('preservative-fixed'), by dividing samples in half upon receipt by the virology laboratory for the first 50 study participants. Preservative-fixed samples were prepared using the Abbott multicollect specimen collection kit and the Roche Cobas PCR urine specimen collection kit, according to manufacturers' instructions. The stability of 
neat and preservative-fixed urine samples was compared by HR-HPV testing using both assays within 48 hours, 1 week and 1 month of receipt. The ct at which HR-HPV and the internal beta globin control was detected was compared in the same samples tested after different time intervals, to determine the stability of DNA in neat and preservative-fixed urine samples. Although the receipt of neat urine samples and their rapid fixation and/or refrigeration within 4 hours of collection is not compatible with urine self-collection at home and postal delivery to the laboratory, this study sought to explore the potential for urinary HR-HPV detection under optimal processing and transportation conditions, to determine proof-of-principle that further studies are warranted.

\section{Statistical analysis}

HPV detection rates were calculated for each assay and sample type. Concordance between the different samples for HR-HPV positivity was determined using Cohen's kappa statistic. Agreement was slight $(\kappa<0.20)$, weak $(\kappa=0.21-0.40)$, moderate $(\kappa=0.41-0.60)$, substantial $(\kappa=0.61-0.80)$, near perfect $(\kappa=0.81-0.99)$ or perfect $(\kappa=1.00)$. The sensitivity of HR-HPV positivity for CIN2+ detection was compared between urine, vaginal and cervical samples. Sensitivity was calculated as the number of true positives divided by the sum of the number of true positives plus false negatives.

\section{Patient and public involvement}

Patients and the public were not involved in designing or conducting this research. We will work with patient support groups and forums, including the Eve Appeal and Jo's Cervical Cancer Trust, to help disseminate the results of this study.

\section{RESULTS}

\section{Study participants}

Of 127 eligible women, 104 took part in the study (figure 1). Their median age range and distribution reflected that of routine colposcopic practice, with $40 \%$ between 25 and 29 years of age. Non-participants had similar demographics to those who did agree to the study. The reasons for non-participation are shown in figure 1 . Triple matched samples were not available for 17 women (no vaginal sample $\mathrm{n}=8$; no cervical sample $\mathrm{n}=5$; no urine sample $n=1$; only urine sample $n=2$; only cervical sample $\mathrm{n}=1$ ).

\section{Optimisation of urine sample processing for HR-HPV testing}

A comparison was made between neat and preservative-fixed urine for HR-HPV detection using both ART and $\mathrm{RC}$ assays for the first 50 study participants. There were 44 matched neat and preservative-fixed urine sample pairs available for the ART assay. Two failed the internal beta globin control and were excluded from further analysis. A ct) cut-off of $\leq 32$ was used to define HR-HPV positivity. table 1 demonstrates good concordance between

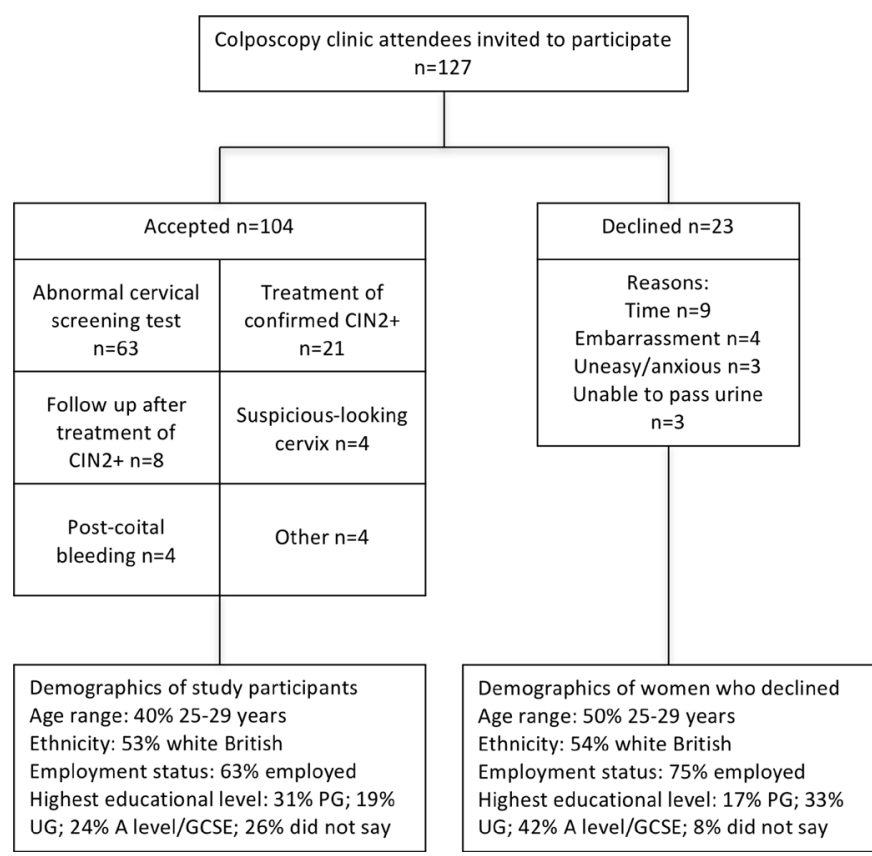

Figure 1 Demographics of study participants and reasons for declining participation. CIN2+, cervical intraepithelial neoplasia grade 2+; GCSE, General Certificate of Secondary Education; PG, postgraduate; UG, undergraduate.

neat and preservative-fixed urine samples $(37 / 42,88 \%$, $\mathrm{k}=0.76,95 \%$ CI 0.56 to 0.96 ).

Repeat testing of the 47 preservative-fixed urine samples approximately 1 month later (mean 33 days) showed no reduction in stability of HR-HPV DNA, with almost identical ct values, as shown in online supplementary figure 1 (mean ct 25.2 for samples tested within 48 hours versus 25.1 for samples tested at 1 month). This was true when all HR-HPV types, HPV 16/18 and HR-HPV other were considered separately (data not shown).

There were 40 matched neat and preservative-fixed urine sample pairs available for the RC assay. Eighteen of the neat but none of the preservative-fixed urine samples, which had been stored for up to 1 week prior to testing, failed the internal beta globin control and were excluded from further analysis. Thus there were 22 matched urine sample pairs available for testing with the $\mathrm{RC}$ assay. A ct of $\leq 40$ was used to define HR-HPV positivity. table 1 shows good concordance between neat and preservative-fixed urine samples $(20 / 22,90 \%, \kappa=0.79,95 \%$ CI 0.52 to 1.0$)$. Based on these data and the apparent instability of neat urine samples over time, all subsequent urine samples were fixed with preservative upon receipt in the lab, and neat urine samples were not considered further.

\section{HR-HPV detection in urine, vaginal and cervical samples ART assay}

From the 104 women, 79 had valid HR-HPV results from matched urine, vaginal and cervical samples for the ART assay. Triple samples were not available for 17 women and invalid results were obtained for one or more sample for a further eight women (seven vaginal samples and one cervical sample). There were no invalid results for urine 
Table 1 Comparison of neat versus preservative-fixed urine using the Abbott RealTime (ART) assay and the Roche Cobas $4800(\mathrm{RC})$ assay for high-risk human papillomavirus detection

\begin{tabular}{lccc}
\hline & $\begin{array}{l}\text { Neat } \\
\text { positive }\end{array}$ & $\begin{array}{l}\text { Neat } \\
\text { negative }\end{array}$ & Total \\
\hline ART assay & & & \\
\hline Preservative positive & 19 & 3 & 22 \\
\hline Preservative negative & 2 & 18 & 20 \\
\hline Total & 21 & 21 & 42 \\
$R C$ assay & & & \\
Preservative positive & 14 & 2 & 16 \\
\hline Preservative negative & 0 & 6 & 6 \\
\hline Total & 14 & 8 & 22 \\
\hline
\end{tabular}

samples. Directed cervical biopsies were taken from 66/79 $(84 \%)$ of these women because of abnormal cytology. Histological analysis classified the biopsies within normal limits $(31 / 66,47 \%)$, CIN1 $(17 / 66,26 \%)$, CIN2 (10/66, $15 \%)$ and CIN3 (8/66, 12\%).

HR-HPV was detected in 48/79 (61\%), 54/79 (68\%) and $56 / 79(71 \%)$ of urine, vaginal and cervical samples, respectively. HPV 16/18 was detected in 21/79 (27\%), 24/79 (30\%) and 23/79 (29\%). Concordance between the three sample types was found in $62 / 79(79 \%)$, as shown in table 2. According to the manufacturer's cut-off of $32 \mathrm{ct}$, the sensitivity for detecting CIN2+ was 16/18 (89\%) for both cervical and vaginal samples and 15/18 $(83 \%)$ for urine samples. Concordance between urine and cervical samples was $82 \%(65 / 79 ; \kappa=0.61 ; 95 \%$ CI 0.43 to 0.79 ) and between vaginal and cervical samples was $90 \%(71 / 79 ; \kappa=0.76 ; 95 \%$ CI 0.61 to 0.92$)$. Only one CIN2+ lesion was 'missed' from the eight women with invalid results by the ART assay (a vaginal sample).

Table 2 High-risk human papillomavirus detection in preservative-fixed urine, vaginal and cervical samples by the Abbott RealTime (ART) assay and the Roche Cobas 4800 $(\mathrm{RC})$ assay

\begin{tabular}{lllll}
\multicolumn{2}{l}{ Cervical positive } & & \multicolumn{2}{l}{ Cervical negative } \\
\cline { 1 - 2 } $\begin{array}{l}\text { Vaginal } \\
\text { positive }\end{array}$ & $\begin{array}{l}\text { Vaginal } \\
\text { negative }\end{array}$ & & $\begin{array}{ll}\text { Vaginal } \\
\text { positive }\end{array}$ & $\begin{array}{l}\text { Vaginal } \\
\text { negative }\end{array}$
\end{tabular}

\begin{tabular}{lcccr}
\hline$A R T$ & & & & \\
Urine positive & $43^{*}$ & 2 & 2 & 1 \\
Urine negative & $8 \dagger$ & 3 & 1 & $19 \ddagger$
\end{tabular}

$R C$

\begin{tabular}{lrrrr} 
Urine positive & $44 \S$ & 0 & 1 & 0 \\
Urine negative & 4 & 0 & 1 & $16 \rrbracket$ \\
\hline
\end{tabular}

*9 CIN2, 6 CIN3.

†1 CIN3.

$\neq 1 \mathrm{CIN} 2,1 \mathrm{CIN} 3$.

§8 CIN2, 7 CIN3.

ๆ1 CIN2, 1 CIN3.
A comparison of the ct values for detection of the internal beta globin control demonstrated lower DNA concentrations in urine compared with vaginal and cervical samples (figure 2A). The mean ct value for urine, vaginal and cervical samples was 25.6, 20.9 and 23.1, respectively, with similar results for samples which showed positive results for HPV 16/18 or HPV other (25.3, 20.6 and 20.9, respectively) (figure $2 \mathrm{~B}$ ).

RC assay

From the 104 women, 66 had valid HR-HPV results from matched urine, vaginal and cervical samples for the RC assay. Triple samples were not available for 17 women, there was insufficient volume for the RC assay for eight cervical and three urine samples, and invalid results were obtained for one or more sample for 10 women (two vaginal and 10 cervical samples). There were no invalid results for urine samples. Cervical biopsies were taken for $54 / 66(82 \%)$ of these women and histological analysis classified these as normal (24/54, 44\%), CIN1 (14/54, 26\%), CIN2 $(8 / 54,15 \%)$ and CIN3 $(8 / 54,15 \%)$.

HR-HPV was detected in 45/66 (68\%), 50/66 (76\%) and $48 / 66(73 \%)$ of urine, vaginal and cervical samples, respectively. HPV 16/18 was detected in 16/66 (24\%), 21/66 (32\%) and 19/66 (29\%). Concordance between the three sample types was found in 60/66 (91\%), as shown in table 2. According to the manufacturer's cut-off of $40 \mathrm{ct}$, the sensitivity for detecting CIN2+ was 15/17 $(88 \%)$ for all three sample types. Concordance between urine and cervical samples was $92 \%(61 / 66, \kappa=0.82$, $95 \% \mathrm{CI} 0.67$ to 0.97 ) and between vaginal and cervical samples was $97 \%(64 / 66, \kappa=0.77,95 \%$ CI 0.60 to 0.92$)$. No CIN2+ lesions were 'missed' among women with invalid results by the RC assay.

A comparison of the mean ct values for detection of the internal beta globin control again demonstrated lower DNA concentrations in urine (figure 2C). The mean ct values for urine, vaginal and cervical samples were 31.7 , 27.5 and 29.9, respectively, with similar findings with concordant positive results for HPV 16/18 or HPV other (33.6, 28.1 and 27.7, respectively) (figure 2D).

\section{Acceptability of urine, vaginal and cervical sampling for cervical screening}

A total of 98 women answered the acceptability questionnaire (online supplementary appendix 1). Most of the participants had heard about HPV before (yes, 73; no, 17; do not know, 1; not answered, 7). Participants were confident about providing self-obtained vaginal and urine samples for HPV testing and were not excessively concerned about the accuracy of the test in these samples (figure 3). In general, women were more confident about providing a urine sample than a vaginal sample for HPV testing. When asked which sample they would prefer to provide, more women chose urine (39) than either vaginal (32) or cervical samples (17) (no preference, 1; not answered, 9). 


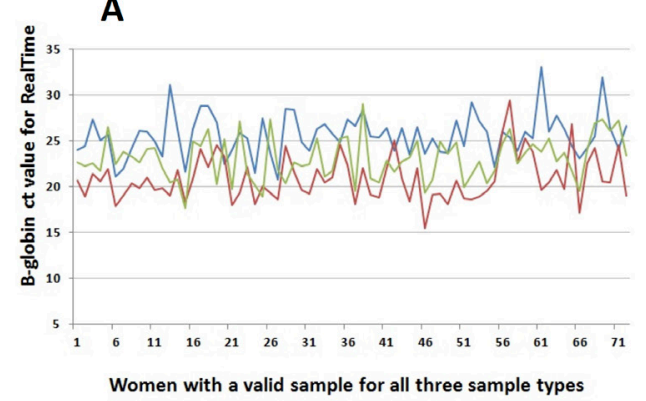

C

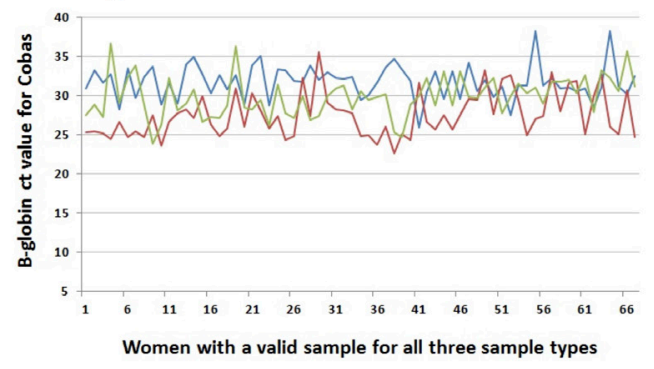

B

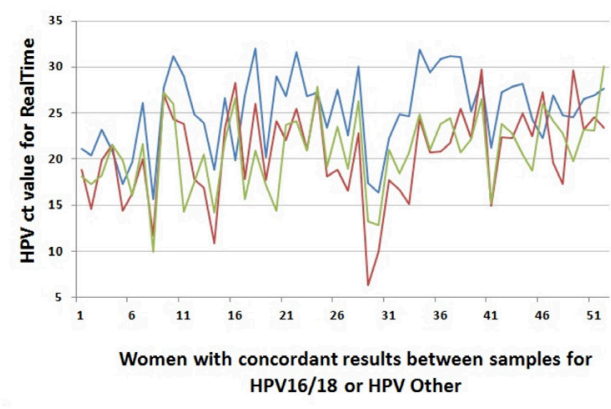

D

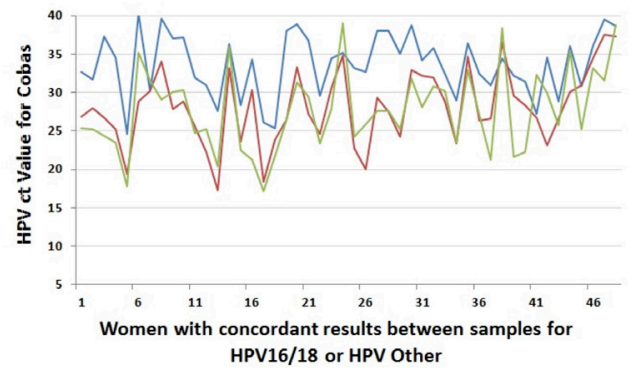

Figure 2 A comparison of the mean ct values for detection of the internal beta globin control and HR-HPV in urine, vaginal and cervical samples using the ART $(A, B)$ and $R C$ assays $(C, D)$. ct, cycle threshold; HPV, human papillomavirus.

\section{DISCUSSION}

This study found good concordance for HR-HPV detection between preservative-fixed urine, vaginal and cervical samples among women attending a colposcopy clinic. Urine showed similar, although slightly inferior, sensitivity to matched vaginal and cervical samples for CIN2+ detection using both ART and RC assays. Cervical screening by urinary and vaginal HR-HPV detection was considered acceptable by women.

This was an exploratory study of women attending the colposcopy clinic for the management of abnormal cervical screening results or a suspicious-looking cervix. Forty per cent of the participants were under 30 years of age. The prevalence of HR-HPV was therefore sufficiently

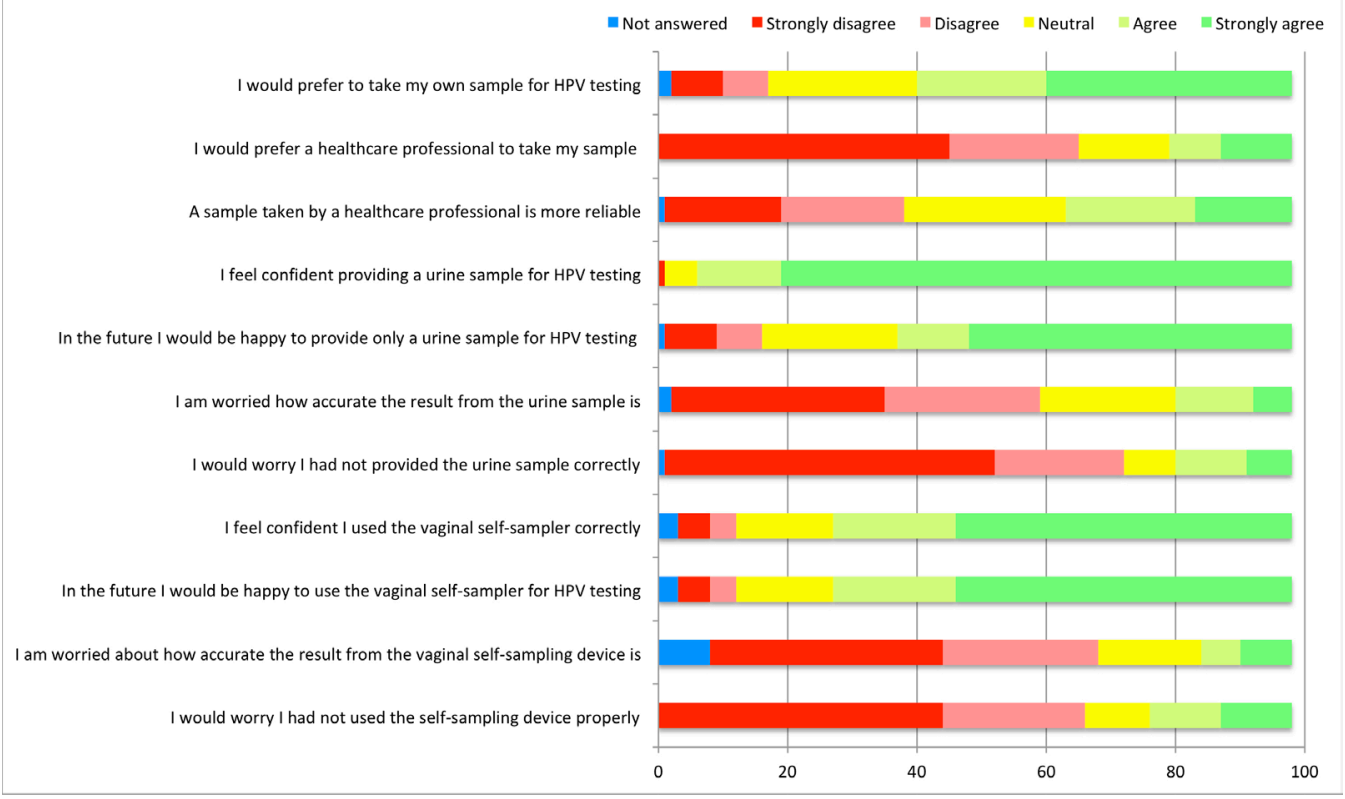

Figure 3 The acceptability of urine and vaginal self-obtained samples for cervical screening. 
high to allow comparison between the three sampling methods. Matched urine, vaginal and cervical samples taken at the same visit and immediately prior to colposcopic assessment, eliminated the possibility that HPV had been acquired or cleared between tests. The order of obtaining matched samples meant that cross-contamination was unlikely. We found that preservative-fixed urine was suitable for HR-HPV detection by both ART and $\mathrm{RC}$ assays within 48 hours and up to 1 month following its receipt. The consistent results showed that preservative-fixed urine can be handled in the same way as cervical samples without compromising analytical sensitivity.

The relatively small size of the study means that urine testing needs to be fully validated in larger cohorts. The colposcopy clinic setting facilitated an assessment of the analytical sensitivity of urinary HR-HPV testing but the true value of urine-based cervical screening is likely to be its ability to improve cervical screening uptake by non-attenders. The prevalence of HR-HPV is much lower in the general population, which could be expected to affect the sensitivity and negative predictive value of the test. Studies designed to evaluate the accuracy and clinical utility of urinary HR-HPV detection in the primary cervical screening population are therefore required. ${ }^{11}$ Urinary self-sampling was acceptable to women attending colposcopy clinic; those who declined participation did so for other reasons. An important limitation of this study is that self-sampling took place before colposcopy, which may have affected women's confidence in the accuracy of self-sampling. The acceptability of urinary self-sampling to cervical screening non-attenders remains unclear, as does the ability of urinary HR-HPV detection to improve uptake. Defining its acceptability in 'hard to reach' groups is challenging but crucial to its further development as an alternative test for cervical screening. Forty per cent of our participants were under 30 years of age, a group of women that stands to benefit most from strategies to improve uptake, given their declining participation in cervical screening programmes. ${ }^{5}$

Previous studies have looked at urine sampling techniques ${ }^{12}{ }^{13}$ but few have reported the optimisation of urine processing and storage for HR-HPV detection. Here we show that preservative-fixed urine has improved stability and can be stored prior to testing for much longer than neat urine, which rapidly deteriorates after 48 hours. This may explain the improved clinical performance of urinary HR-HPV reported here compared with studies that tested neat urine. ${ }^{8}$ In total, $45 \%$ of neat urine samples tested on the RC assay at 1 week were invalid compared with only $2 \%$ of samples tested within 48 hours of collection by ART assay. This can be explained by an increase in PCR inhibitors over time. Preservative-fixed urine stored at $4^{\circ} \mathrm{C}$ showed only one invalid result using the ART assay among 104 urine samples tested within 48 hours, and there were no invalid results using the RC assay among 98 urine samples tested between 7 and 14 days. Repeat testing at 1 month showed consistent results. While the requirement to preserve urine samples challenges the ideal scenario of home collection and postage of urine samples to the laboratory, it would not preclude self-collection within Primary Care, where preservative can be safely added shortly afterwards.

The analytical and clinical sensitivity of the RC and ART assays for cervical HR-HPV detection are well documented, ${ }^{14-16}$ and both assays are approved for primary HPV screening, HPV triage of minor cytological abnormalities and test of cure in the NHS Cervical Screening Programme. Neither assay is currently Conformité Européenne (CE) marked for urinary testing and while the data in this pilot study are consistent with published studies, more work is needed to define viral load thresholds of clinical utility for the detection of CIN2+ ${ }^{17} 18$ Raising the threshold of the ART assay above 32 ct (manufacturer's cut-off) did pick up four more positive urine samples but did not identify the single undetected CIN2+, which suggests that the manufacturer's cut-offs are appropriate for urine samples. The analytical sensitivity for HR-HPV in urine, vaginal and cervical samples were similar though a lower proportion of HR-HPV was detected in urine samples compared with vaginal samples.

Barriers to cervical screening include embarrassment, fear, inconvenience and the view held by some women that they are not at risk. ${ }^{4}$ Offering an alternative means of cervical screening can encourage non-attenders to be screened, either by 'nudging' them to undergo cervical sampling or by facilitating self-sampling at home. ${ }^{5}$ Urinebased testing is already widely used for the detection of chlamydia and is generally trusted and acceptable to women. The disadvantage of self-testing is that reflex cytology is not possible in the event of a positive HR-HPV test, however, data suggest that women who screen positive for HR-HPV are highly likely to attend for cervical cytology. ${ }^{19}$ Urine-based testing may also be cheaper than standard cervical screening tests because it does not require an appointment, a practitioner or any special equipment and it lends itself to high throughput testing. Thus it could also be suitable in resource poor settings where the infrastructure required to underpin screening by cervical cytology is lacking.

\section{CONCLUSIONS}

Urinary HR-HPV testing has good sensitivity for the detection of CIN2+ in a colposcopy clinic cohort. Our study suggests that vaginal HR-HPV testing is slightly more sensitive than urine HR-HPV testing, however urine self-sampling may be more acceptable to cervical screening non-attenders than vaginal self-sampling. Future studies are warranted to assess the performance and acceptability of urine self-sampling in comparison with vaginal self-sampling in the general screening population.

\section{Author affiliations}

${ }^{1}$ Clinical Virology Department, Manchester Royal Infirmary, Manchester University NHS Foundation Trust, Manchester, UK

${ }^{2}$ Gynaecological Oncology Research Group, Division of Cancer Sciences, University of Manchester, Faculty of Biology, Medicine and Health, Manchester, UK 
${ }^{3}$ Department of Obstetrics and Gynaecology, St Mary's Hospital, Manchester University NHS Foundation Trust, Manchester Academic Health Science Centre, Manchester, UK

\section{Twitter @DrEmmaCrosbie}

Acknowledgements The authors would like to thank all the women who participated in this study and donated their samples and data for research. We would like to thank Mrs Linsey Nelson for her support with obtaining all the necessary regulatory approvals and for data management.

Contributors AS performed the laboratory analyses, data interpretation and wrote the first draft of the manuscript. SF and KB recruited and sampled the study participants and contributed to data interpretation. HCK and EJC designed the study and contributed to data interpretation and manuscript preparation. All authors reviewed the final version of the manuscript.

Funding EJC is a National Institute for Health Research (NIHR) Clinician Scientist (NIHR-CS-012-009) and is supported by the NIHR Manchester Biomedical Research Centre (IS-BRC-1215-20007). This research received no specific grant from any funding agency in the public, commercial or not-for-profit sectors.

Competing interests HCK is Chair of the Advisory Committee for Cervical Screening (PHE), but the views expressed in this manuscript are those of the author and not Public Health England.

Patient consent for publication Not required.

Ethics approval The study was approved by the North West Research Ethics Committee (15/NW/0218) and all women gave written, informed consent to take part.

Provenance and peer review Not commissioned; externally peer reviewed.

Data sharing statement Data are available and will be shared upon reasonable request.

Open access This is an open access article distributed in accordance with the Creative Commons Attribution Non Commercial (CC BY-NC 4.0) license, which permits others to distribute, remix, adapt, build upon this work non-commercially, and license their derivative works on different terms, provided the original work is properly cited, appropriate credit is given, any changes made indicated, and the use is non-commercial. See: http://creativecommons.org/licenses/by-nc/4.0/.

\section{REFERENCES}

1. Crosbie EJ, Einstein $\mathrm{MH}$, Franceschi S, et al. Human papillomavirus and cervical cancer. Lancet 2013;382:889-99.

2. Kitchener HC, Denton K, Soldan K, et al. Developing role of HPV in cervical cancer prevention. BMJ 2013;347:f4781.

3. Ronco G, Dillner J, Elfström KM, et al. Efficacy of HPV-based screening for prevention of invasive cervical cancer: follow-up of four European randomised controlled trials. Lancet 2014;383:524-32.
4. Waller J, Bartoszek M, Marlow L, et al. Barriers to cervical cancer screening attendance in England: a population-based survey. $J$ Med Screen 2009;16:199-204.

5. Kitchener HC, Gittins M, Rivero-Arias O, et al. A cluster randomised trial of strategies to increase cervical screening uptake at first invitation (STRATEGIC). Health Technol Assess 2016;20:1-138.

6. Verdoodt F, Jentschke M, Hillemanns P, et al. Reaching women who do not participate in the regular cervical cancer screening programme by offering self-sampling kits: a systematic review and meta-analysis of randomised trials. Eur J Cancer 2015;51:2375-85.

7. Polman NJ, Ebisch RMF, Heideman DAM, et al. Performance of human papillomavirus testing on self-collected versus cliniciancollected samples for the detection of cervical intraepithelial neoplasia of grade 2 or worse: a randomised, paired screen-positive, non-inferiority trial. Lancet Oncol 2019;20:229-38.

8. Stanczuk G, Baxter G, Currie $\mathrm{H}$, et al. Clinical validation of hrHPV testing on vaginal and urine self-samples in primary cervical screening (cross-sectional results from the Papillomavirus Dumfries and Galloway-PaVDaG study). BMJ Open 2016;6:e010660.

9. Pathak N, Dodds J, Zamora J, et al. Accuracy of urinary human papillomavirus testing for presence of cervical HPV: systematic review and meta-analysis. BMJ 2014;349:g5264

10. Kitchener HC, Owens GL. Urine Testing for HPV. A promising screening option that deserves further evaluation. $B M J$ 2014;349:g5542.

11. Blake DA, Crosbie EJ, Kitson S. Urinary HPV testing may offer hope for cervical screening non-attenders. BJOG 2017:124:1364.

12. Johnson DJ, Calderaro AC, Roberts KA. Variation in nuclear DNA concentrations during urination. J Forensic Sci 2007;52:110-3.

13. Vorsters A, Van den Bergh J, Micalessi I, et al. Optimization of HPV DNA detection in urine by improving collection, storage, and extraction. Eur J Clin Microbiol Infect Dis 2014;33:2005-14.

14. Heideman DA, Hesselink AT, Berkhof J, et al. Clinical validation of the cobas $4800 \mathrm{HPV}$ test for cervical screening purposes. J Clin Microbiol 2011;49:3983-5.

15. Poljak M, Ostrbenk A, Seme K, et al. Comparison of clinical and analytical performance of the Abbott Realtime High Risk HPV test to the performance of hybrid capture 2 in population-based cervical cancer screening. J Clin Microbiol 2011;49:1721-9.

16. Moss SM, Bailey A, Cubie $\mathrm{H}$, et al. Comparison of the performance of HPV tests in women with abnormal cytology: results of a study within the NHS cervical screening programme. Cytopathology 2015;26:373-80.

17. Daponte A, Pournaras S, Mademtzis I, et al. Evaluation of high-risk human papillomavirus types PCR detection in paired urine and cervical samples of women with abnormal cytology. J Clin Virol 2006;36:189-93.

18. Daponte A, Tsezou A, Oikonomou P, et al. Use of real-time PCR to detect human papillomavirus-16 viral loads in vaginal and urine selfsampled specimens. Clin Microbiol Infect 2008;14:619-21.

19. Szarewski A, Cadman L, Mesher D, et al. HPV self-sampling as an alternative strategy in non-attenders for cervical screening - a randomised controlled trial. Br J Cancer 2011:104:915-20. 